\title{
DYNAMIC PROPERTIES AND FATIGUE FAILURE OF AIRCRAFT COMPONENT
}

\section{S.Kuznetsov,}

Postgraduate student Aviation Institute of Riga Technical University, Latvia

\section{E.Ozolinsh,}

Ing.Sc.Dr. Aviation Institute of Riga Technical University, Latvia

\section{I.Ozolinsh,}

Ing.Sc.Dr. Aviation Institute of Riga Technical University, Latvia

\section{I.Pavelko}

Ass,Prof. Aviation Institute of Riga Technical University, Latvia

\section{V.Pavelko}

Prof. Aviation Institute of Riga Technical University, Latvia

Keywords: aircraft component, dynamics, non-classic constraint, fatigue damage

\begin{abstract}
The basic purpose of given article is consideration of the problems connected with application of a method of concentrated weights in the tasks of mechanical systems dynamics at non-classic internal and external constraints. The method of the concentrated weights is convenient means of the analysis of dynamic properties of elastic mechanical systems. It has relative simplicity of definition of the parameters of equivalent discrete system, the clearness of computing algorithms and provides comprehensible accuracy of definition of the lowest natural frequencies. Doubtless advantage of a method is convenience of modeling of non-classic constraints of fastening and internal constraints between elements of complex systems. Such problems arise at the decision of practice tasks of the analysis of dynamics of real systems. The method is used for the analysis of vibra-
\end{abstract}


tions of a beam with variable parameters at presence of elastic supporting of the beam and the attached additional concentrated weight. A result of dynamic analysis has a good correlation with the fatigue damages observed at fatigue test of a beam.

\section{Introduction}

This article is connected with 6FP Euro project AISHA. The basic purpose of the project - development of the continuous monitoring system of a technical condition integrated into a structure. Progressive methods and means of the control over use of ultrasonic technology are developed. In thin-walled structures it uses properties of elastic Lamb waves. The final stage provides carrying out of full-scale fatigue tests on components of real aviation structures for demonstration of working capacity and efficiency of methods and means of the non-destructive testing. The object of testing is the helicopter MI-8 tail beam (Figure 1). It is Al alloy typical aircraft component.

At planning experiment there are the problems with a choice of an optimum mode of the tests combining realization of demanded distribution of stresses, duration of tests and relative simplicity of excitation of mechanical load. In this connection there was a necessity of the analysis of dynamic characteristics of the testing object.

There is a plenty of methods of the dynamic analysis of mechanical system [1-4]. Exact analytical methods have the limited application and, mainly, their role is the

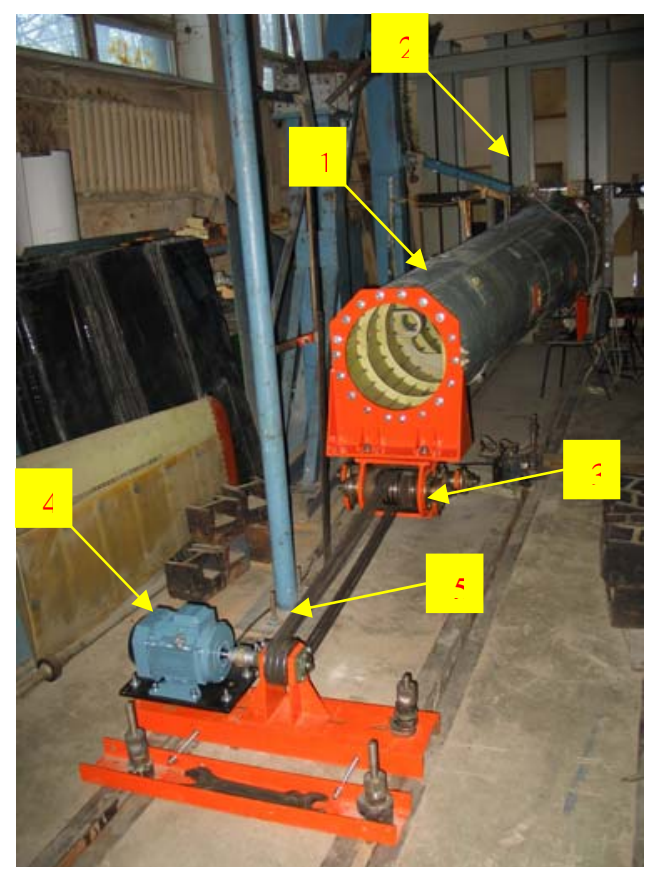

Figure 1. Stand of dynamic testing of the helicopter MI-8 tail beam ( 1 - the tail beam, 2support, 3- mechanical vibrator, 4 - motor, 5 - V-belt gear) 
reference decisions for an estimation of accuracy of decisions by the approximated methods. Among the last the visible place is occupied by Ritz's methods [5], various versions of energetic methods [6], and also methods of replacement of system with continuously parameters by system with the limited number of the concentrated weights [6-8]. Universal numerical method of finite elements (FEM) is also some one of this group method [9].

There are many methods of the dynamic behavior analysis of complex elastic structure and usually special estimation of accuracy by comparison with precise analytical solution shows the rational selection of model parameters allows obtaining of acceptable result of simulation. But modeling of boundary conditions is often no adequate. Therefore real experimental data is unacceptable difference from results of simulation in similar cases.

In presented research there are two aims. First of them is the method of the concentrated weights application for definition of dynamic characteristics of real aircraft component with continuously distributed weight. Second (and it is main aim) is correct simulation of non-classic internal and external constraints at application of a method of concentrated weights.

\section{The Method of Concentrated Weights}

In connection with the relatively big length of a beam in comparison with diameter of its cross-section the beam schematization of such structure is admissible at the analysis of its dynamic characteristics. The equation of movement in this case looks like

$m(z) \frac{\partial^{2} v}{\partial t^{2}}+\frac{\partial^{2}}{\partial z^{2}}\left(E J_{x} \frac{\partial^{2} v}{\partial z^{2}}\right)=q(z, t)$

where $\boldsymbol{v}(\boldsymbol{z}, \boldsymbol{t})$ is a deflection of a beam, $\boldsymbol{m}(\boldsymbol{z})$ is the intensity of the weight distribution, $\boldsymbol{E}_{\boldsymbol{x}}$ is bending stiffness of the cross-sections of a beam, and $\boldsymbol{q}(\boldsymbol{z}, \boldsymbol{t})$ is intensity of the distributed external load. If $\boldsymbol{q}(\boldsymbol{z}, \boldsymbol{t}) \equiv \boldsymbol{0}$, the free movement without participation of external forces is realized.

Generally, if the mass and stiffness of the cross-sections is distributed nonuniformly, the analytical decision cannot be received. Therefore the approximated method of the concentrated weights is used for the purpose of this research. For definition of natural frequencies and forms the actual beam with continuously distributed weight must be replaced by a weightless beam with the same bending stiffness, but with finite number of the concentrated weights in finite number of nods $(\boldsymbol{k}+1)$. For this purpose the beam has been sheared into finite number $\boldsymbol{k}$ parts. For each of them weight $\boldsymbol{M}_{\boldsymbol{i}}$ and its center coordinate $\boldsymbol{z}_{\boldsymbol{i c}}$ was defined and then it was distributed between nods (i-1) and $\boldsymbol{i}$ those are finite nods of the part. From a 
condition of static equivalence there are the next expressions

$M_{i}=\Delta M_{i-1}+\Delta M_{i}$

$M_{i} \cdot z_{i c}=\Delta M_{i-1} \cdot z_{i-1}+\Delta M_{i} \cdot z_{i}=\int_{z_{i-1}}^{z_{i}} z_{i} \cdot m(z) d z$

where $\boldsymbol{\Delta} \boldsymbol{M}_{\boldsymbol{i}-1}$ and $\boldsymbol{\Delta} \boldsymbol{M}_{\boldsymbol{i}}$ are the lots of weight of the part, attached to units (i-1) and $\boldsymbol{i}$ accordingly.

If the beam is sheared into enough plenty of parts within the limits of one part distribution of weight can be accepted linear and then last two equations can be written down so

$M_{i}=\Delta M_{i}+\Delta M_{i-1} \cong \frac{m_{i}+m_{i-1}}{2} \cdot \Delta z_{i}$

$M_{i} z_{i c}=\Delta M_{i-1} z_{i-1}+\Delta M_{i} z_{i} \cong \frac{m_{i} z_{i}+m_{i-1} z_{i-1}}{2} \cdot \Delta z_{i}$

or in the dimensionless form

$\bar{M}_{i}=\Delta \bar{M}_{i}+\Delta \bar{M}_{i-1} \cong \frac{\bar{m}_{i}+\bar{m}_{i-1}}{2} \cdot \Delta \bar{z}_{i}$,

$\bar{M}_{i} \cdot \bar{z}_{i c}=\Delta \bar{M}_{i-1} \cdot \bar{z}_{i-1}+\Delta \bar{M}_{i} \cdot \bar{z}_{i} \cong \frac{\bar{m}_{i} \cdot \bar{z}_{i}+\bar{m}_{i-1} \cdot \bar{z}_{i-1}}{2} \cdot \Delta \bar{z}_{i}$

where $\Delta \bar{M}_{i}=\Delta M_{i} /\left(m_{0} l\right), \quad \Delta \bar{z}_{i}=\Delta z_{i} / l$

As a result relative weights can be expressed in the dimensionless form so

$\Delta \bar{M}_{i}=\bar{M}_{i} \cdot \frac{\bar{z}_{i}-\bar{z}_{i c}}{\Delta \bar{z}_{i}}, \quad \Delta \bar{M}_{i-1}=\bar{M}_{i} \cdot \frac{\bar{z}_{i c}-\bar{z}_{i-1}}{\Delta \bar{z}_{i}} \ldots \ldots \ldots \ldots \ldots \ldots \ldots \ldots \ldots \ldots \ldots \ldots \ldots \ldots \ldots$

2)

Where

$\bar{z}_{i c}=\frac{\bar{m}_{i} \cdot \bar{z}_{i}+\bar{m}_{i-1} \cdot \bar{z}_{i-1}}{2 M_{i}} \cdot \Delta \bar{z}_{i}$ 
It is obvious, that the total relative weight in the nod $\boldsymbol{i}$ is $\overline{\boldsymbol{m}}_{\boldsymbol{i}}=\overline{\boldsymbol{M}}_{\boldsymbol{i}}+\overline{\boldsymbol{M}}_{\boldsymbol{i + 1}}$.

On the basis of a principle of superposition the displacement $\boldsymbol{\Delta} \boldsymbol{i}$ in the nod $\boldsymbol{i}(\boldsymbol{i}=\mathbf{0}$, $1, \ldots k)$ as result of the action of system of the vertical forces $\boldsymbol{P}_{j}$ concentrated in all nods, is defined by the following sum

$$
\Delta_{i}=\sum_{j=1}^{n} \delta_{i j} \cdot P_{j}=-\sum_{j=1}^{n} \delta_{i j} \cdot \boldsymbol{m}_{j} \cdot \Delta_{j}^{\prime \prime}
$$

where $\boldsymbol{\delta}_{i j}$ is the factor of elastic compliance.

In the second part of equation (3) the force $\boldsymbol{P}_{j}$ is replaced by the inertial force that appears in case of natural vibrations. These vibrations are harmonious with frequency $\omega$. Therefore

$$
\sum_{j=1}^{n} \delta_{i j} \cdot m_{j} \Delta_{j}^{\prime \prime}+\Delta_{i}=0 \quad \Delta_{i}=A_{i} \cdot \operatorname{Sin} \omega t
$$

where $\boldsymbol{A}_{\boldsymbol{i}}$ is amplitude of vibration of the nod $\boldsymbol{i}$.

As a result for definition of amplitudes of free vibrations the system of $\boldsymbol{k}$ the linear homogeneous algebraic equations turns out

$$
\delta_{i 1} m_{1} A_{1}+\ldots+\left(-\frac{1}{\omega^{2}}+\delta_{i i} m_{i}\right) A_{i}+\ldots+\delta_{i n} m_{n} A_{n}=0 \ldots(i=1, \ldots, k)
$$

For a greater generality this system of the equations is convenient for writing down in a dimensionless view

$\bar{\delta}_{i 1} \bar{m}_{1} A_{1}+\ldots+\left(-x+\bar{\delta}_{i i} \bar{m}_{i}\right) A_{i}+\ldots+\bar{\delta}_{i n} \bar{m}_{n} A_{n}=0$

where

$$
x=\frac{E J_{x 0}}{m_{0} l^{2}} \cdot \frac{1}{\omega^{2}}, \quad \bar{\delta}_{i j}=\frac{\delta_{i j}}{l^{3} /\left(E J_{x 0}\right)}, \quad m_{j}=\frac{m_{j}}{m_{0} l}
$$

It is known that non-trivial decision of system (5) exists, if the determinant of coefficients of the equations is equal to zero.

$$
\operatorname{Det}\left\{\left[\bar{\delta}_{i j} \bar{m}_{j}\right]-x[I]\right\}=0
$$

Roots $\boldsymbol{x}_{\boldsymbol{k}}$ of the equation (6) define natural frequencies of vibrations. 
$\omega_{k}=\frac{1}{l^{2}} \cdot \sqrt{\frac{E J_{x 0}}{x_{k} m_{0}}}$

After their definition the natural forms can be received too.

Verification of definition of dynamic characteristics by a method of the concentrated weights was carried out calculation for a console beam of constant crosssection and uniformly distributed weight. It is established, if there is $\boldsymbol{k}=\boldsymbol{8} \ldots \mathbf{1 0}$, then at least first two natural frequencies practically coincide with their exact values.

\section{The Analysis of Dynamic Characteristics of a Beam Under Ideal Boundary Conditions}

The method described above leads the numerical analysis of natural frequencies and forms of a beam of the helicopter at perfect conditions of supporting: a jamming at one tip. In Figure 2 the scheme of the test system is presented. The thinwalled tail beam of the helicopter has an elastic attachment 1 to the motionless tip, in the middle part of a beam the additional weight 2 (about $94 \mathrm{~kg}$ ) is attached.

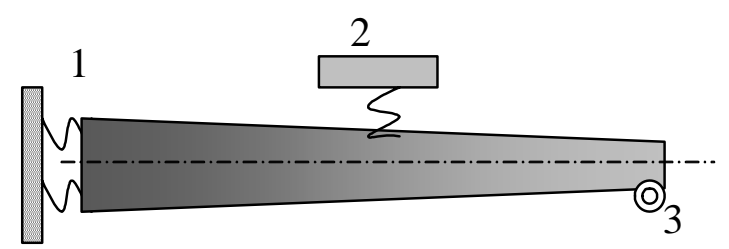

Fig. 2. The beam has elastic support 1 and the mass 2 also has the same kind of connection with beam, 3 is the vibrator

On the free tip of a beam the mechanical vibrator 3 in weight about $50 \mathrm{~kg}$ is installed. If to accept, that connections of a beam with the basis and additional weight with a beam are absolutely rigid, then the relative distribution of weight among nods is in table 1 . Own weight of the beam, the attached weight in an middle zone of a beam and weight of the vibrator consist common weight.

In Fig. 3 results of calculation of first two natural frequencies and forms are shown. In connection with large bending stiffness of a beam and small weight it has high frequencies. The second form of vibrations is obeyed by the requirements of planned tests: the maximal curvature bended axes of a beam so, and the maximal stress take place in the middle part of a beam. 
Table 1. Mass distribution among the nods of system

\begin{tabular}{|l|c|c|c|c|c|c|}
\hline $\begin{array}{c}\text { Weight } \\
\text { number }\end{array}$ & 0 & 1 & 2 & 3 & 4 & 5 \\
\hline $\mathrm{m} / \mathrm{m}_{0}$ & 1 & 0.043 & 0.063 & 0.061 & 0.243 & 0.241 \\
\hline
\end{tabular}

\begin{tabular}{|r|c|c|c|c|c|}
\hline $\begin{array}{c}\text { Weight } \\
\text { number }\end{array}$ & 6 & 7 & 8 & 9 & Total \\
\hline $\mathrm{m} / \mathrm{m}_{0}$ & 0.049 & 0.041 & 0.039 & 0.220 & 1.00 \\
\hline
\end{tabular}



Fig.3. First and second natural form of vibration

\section{The Account of Influence of Non-classic Internal and External Constraints}

Two types of deviations from ideal boundary conditions are examined:

- Elastic fastening;

- An elastic attachment of the additional concentrated weight.

Let the characteristic of the elastic hinge looks like $\boldsymbol{M}_{\boldsymbol{0}}=\boldsymbol{K} \boldsymbol{\theta}$, where $\boldsymbol{K}$ is factor of elastic hinge stiffness, $\boldsymbol{\theta}$ is the angle of turn in the hinge, $\boldsymbol{M}_{\boldsymbol{0}}$ is the moment in the hinge.

At action of single force in nod there is moment $\boldsymbol{M}_{\boldsymbol{0}}=\mathbf{1} \boldsymbol{z}_{j}$ causing rigid turn of a beam about the axis of the hinge $\boldsymbol{\theta}_{j}=\boldsymbol{M}_{\boldsymbol{0}} / \boldsymbol{K}=\boldsymbol{z}_{\boldsymbol{j}} / \boldsymbol{K}$. As a result in section $\boldsymbol{j}$ there will be the additional deflection caused by an elastic compliance of the hinge 
$\delta_{0 i j}=\theta_{j} z_{i}=\frac{z_{i} z_{j}}{K}$

or in the dimensionless form

$\bar{\delta}_{0 i j}=\frac{\delta_{0 i j}}{l^{3} /\left(E J_{x 0}\right)}=\frac{\bar{z}_{i} \bar{z}_{j}}{K l /\left(E J_{x 0}\right)}$

So that to consider influence of elastic compliance hinge fastenings it is necessary to calculate a total compliance $\overline{\boldsymbol{\delta}}_{\Sigma j}=\overline{\boldsymbol{\delta}}_{i j}+\overline{\boldsymbol{\delta}}_{0 i j}$. The subsequent calculation of frequencies and forms remains the same.

The account of elasticity of an attachment of the additional concentrated weight can be shown also to updating a matrix of an elastic compliance. Let the additional weight $\boldsymbol{m}_{\boldsymbol{a}}$ is attached in a nod $\boldsymbol{k}$ by means of elastic connection with stiffness $\boldsymbol{C}$. It is obvious, that connection of additional weight increases number of degrees of freedom and, means, the number of the equations of movement will be equal $\boldsymbol{n}+\boldsymbol{1}$. Thus

$\delta_{i, n+1}=\left\{\begin{array}{l}\delta_{i k}, \ldots \ldots \ldots \ldots \ldots . . . . i \neq k \\ \delta_{k k}+1 / C, \ldots . i=k\end{array}\right.$

Both of the kinds of elastic connections are available in the dynamic system examined in p.3. For the real object prepared for test, the approximated estimation of stiffness of connections has been executed. Their relative values are equal accordingly

$$
\bar{K}=\frac{K}{E J_{x 0} / l}=2.0 \quad \bar{C}=\frac{C}{1 / \delta_{k k}}=1.0
$$

Figure 4 presents results of calculation of first two natural frequencies and forms of a beam in structure of this dynamic system. The natural form value for additional concentrated weight is equal -1.42 . It means the displacement of additional concentrated weight in 1.42 times more than the displacement of free tip of the beam and has opposite phase of vibrations. It is visible, that on the first form of moving of a beam are defined mainly by an angular compliance of a trailer support. The axis of a beam is kept practically direct that testifies about small effect of bending. The second natural frequency and the form in this case is more similar to the first frequency and the form of vibrations at a rigid jamming. Nevertheless this form is much more complex.

The lead analysis shows exclusively strong influence of conditions of fastening of a beam and presence of elastic connections with other objects to dynamic characteristics, in particular, to natural forms and frequencies of vibrations 


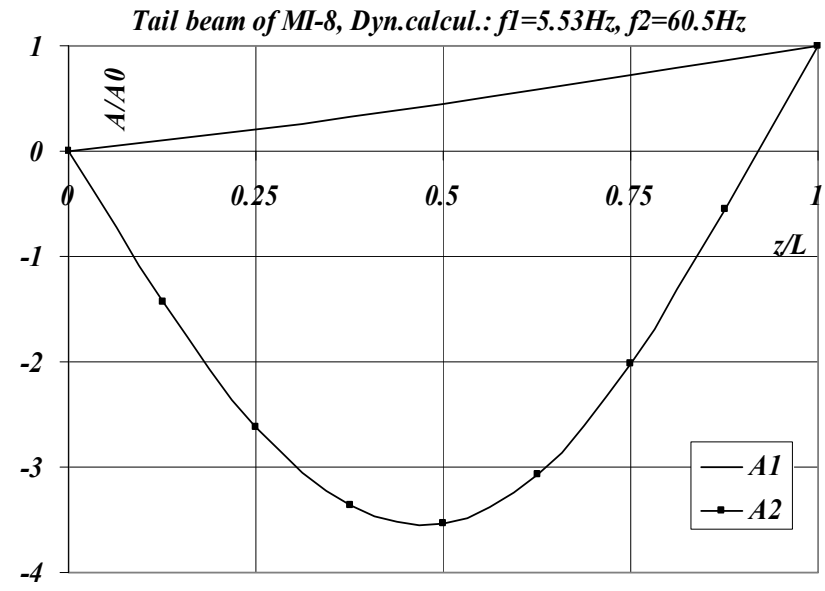

Figure 4. First and second natural frequencies and forms of the tail beam of helicopter at non-classic connections

The estimation of nominal stress of a beam under its form bended axes

Let $\boldsymbol{v}_{\boldsymbol{i}}(\boldsymbol{z})$ is the form $\boldsymbol{i}$ of the bended axes of a beam. Then the bending moment $\boldsymbol{M}(\boldsymbol{z})$ in this section is defined by curvature of this axis in the given section

$M(z)=E J_{x} \frac{d^{2} v_{i}}{d z^{2}}$

and the maximal direct stress are defined under the formula

$$
\sigma_{\max }=\frac{M(z)}{W(z)}
$$

where $\boldsymbol{W}(\boldsymbol{z})$ is the moment of resistance of section.

If amplitude of moving of the free end of a beam to designate $\boldsymbol{A}_{\boldsymbol{0}}$, then the formula (2) of the maximal direct stress can be wrote in next form

$$
\sigma_{\max }=\frac{E J_{x}(z)}{W(z)} \frac{d^{2} v_{i}}{d z^{2}}=\frac{1}{2 l^{2}} E D A_{0} \frac{d^{2} \bar{v}_{i}}{d \bar{z}^{2}}
$$


where $\boldsymbol{J}_{\mathrm{x}}(\mathrm{z})$ is the moment of inertia of section, $\boldsymbol{D}$ and $\boldsymbol{l}$ is external diameter of section and length of a beam, $\boldsymbol{E}$ is the module of elasticity of a material of a beam. The form of vibrations is expressed in the relative form $\overline{\boldsymbol{v}}_{\boldsymbol{i}}=\boldsymbol{v}_{\boldsymbol{i}} / \boldsymbol{A}_{0}$ as a function of relative coordinate of section $\overline{\boldsymbol{z}}=\boldsymbol{z} / \boldsymbol{l}$.

It is obvious the constant $\boldsymbol{\sigma}_{0}=\frac{1}{2 \boldsymbol{l}^{2}} \boldsymbol{E} \boldsymbol{D} \boldsymbol{A}_{0}$ has the same dimension as the stress and its value is defined by the amplitude of moving of the free tip of a beam. As a result

$\bar{\sigma}_{\max }=\frac{\sigma_{\max }}{\sigma_{0}}=\frac{d^{2} \bar{v}_{i}}{d \bar{z}^{2}}$

Thus, the maximal stress distribution along beam longitudinal axis at a bend of a beam under the form $\boldsymbol{i}$ is completely defined by the relative curvature of bended axes. For definition of absolute value of stress it is necessary to set amplitude of moving of the free tip of a beam and to calculate a scale constant $\boldsymbol{\sigma}_{0}$.

In [12] first two natural frequencies and forms of a tail beam of helicopter Mi-8 are obtained at the conditions of supporting those are approximately the same as at dynamic tests. It allows leading an estimation of the maximal direct stress in the sections of a beam. With this purpose the second form presented in the relative form was approximated by a polynomial of the fifth degree. There is enough high accuracy of approximation of the form. However it is known that differentiation of a polynomial can lead to greater mistakes for derivatives. Therefore it was carried out also approximation by a polynomial of higher degree. Comparison of results

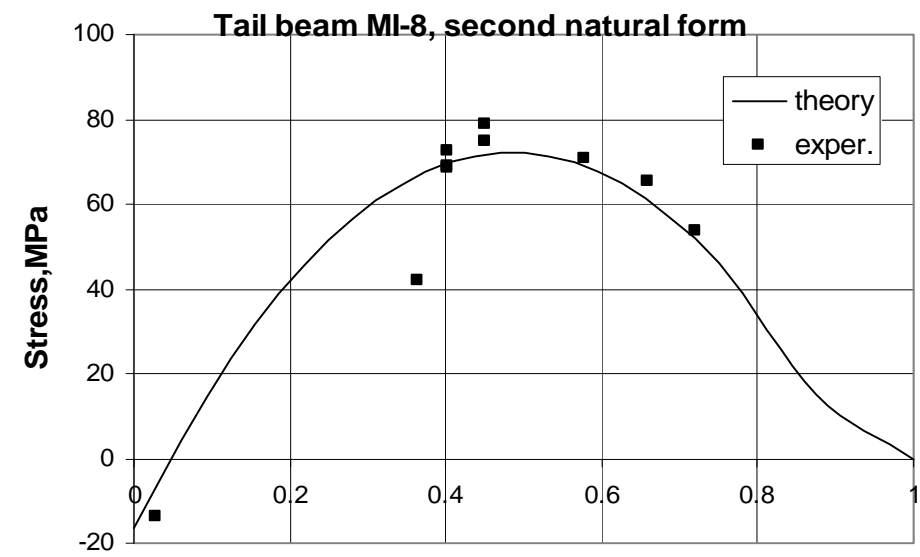

z/L

Figure 5. Comparison theoretical and experimental results for the dvnamir strecs in a skin of tail heam of helirnnter 
really confirms this statement, if to consider the form on all length of a beam. However, significant differences are characteristic for zones of small stress, near to the tips of a beam. In the most intense zone the difference of stresses at the fifth and sixth degrees of a polynomial are insignificant.

In Figure 5 the corrected results of calculation of relative pressure in sections of a beam are presented. It is visible, that the maximum of pressure is approximately on three quarters of length of a beam.

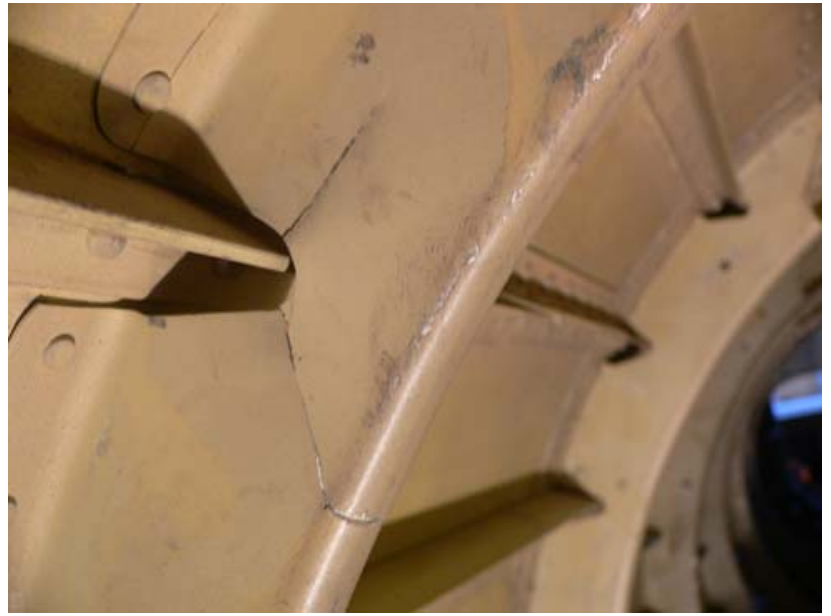

Fig. 6. The fatigue cracks in a frame as a result of dynamic overload induced by additional concentrated weight in middle part of the tail beam

Dynamic tests at frequency of the exciting force equal to the second natural frequency of beam were caused with initiation of fatigue damages to a zone of predicted maximal stresses. The example of a fatigue crack in a frame of a beam is presented on Figure 6.

\section{Conclusions}

The method of the concentrated weights is convenient means of the analysis of dynamic properties of elastic mechanical systems. It has relative simplicity of definition of the parameters of equivalent discrete system, the clearness of computing algorithms and provides comprehensible accuracy of definition of the lowest natural frequencies. Doubtless advantage of a method is convenience of modeling of non-classic constraints of fastening and internal constraints between elements of complex systems. Such problems arise at the decision of practical problems of the analysis of dynamics of real systems. Presented calculation for the real testing system demonstrates very important effect of real boundary conditions 
to dynamic properties of elastic systems.

Acknowledgments This research was induced by the authors' participation in 6FP research project AISHA (Aircraft Integrated Structural Health Assessment). AISHA has brought together major European aircraft manufactures, research and academic institutions in order to provide effective integrated system of continues detection of damages in the aircraft structure.

\section{References:}

1. Timoshenko, S. P., Young, D. H. and Weaver, W., Vibration Problems in Engineering, 4th edn, Wiley, 1974.-444 pp.

2. Tse, E S., Morse, I. E. and Hinkle, R. T., Mechanical Vibrations, Theory and Applications, 2nd edn, Allyn and Bacon, 1983.

3. C. Mei. Differential Transformation Approach for Free Vibration Analysis of a Centrifugally Stiffened Timoshenko Beam.-Journal of Vibration and Acoustics -- April 2006 -- Volume 128, Issue 2, pp. 170-175

4. Francesco Romeo, Angelo Luongo. A Transfer-Matrix-Perturbation Approach to the Dynamics of Chains of Nonlinear Sliding Beams.- Journal of Vibration and Acoustics -- April 2006 -- Volume 128, Issue 2, pp. 190-196

5. Nicole L. Zirkelback and Jerry H. Ginsberg. Ritz Series Analysis of Rotating Shaft System: Validation, Convergence,Mode Functions, and Unbalance Response.-Journal of Vibration and Acoustics, VOLUME 124 - NUMBER $4 \cdot$ OCTOBER 2002, pp.492-501.

6. James, M. L., Smith, G. M., Wolford, J. C. and Whaley, P. W., Vibration of Mechanical and Structural Systems, Harper Row, 1989.

7. Newland, D. E., Mechanical Vibration Analysis and Computation, Longman, 1989.

8. Collar, A. R. and Simpson, A., Matrices and Engineering Dynamics, Ellis Horwood,1987.

9. Huebner, K. H., The Finite Element Method for Engineers, Wiley, 1975.

10. Close, C. M. and Frederick, D. K., Modeling and Analysis of Dynamic Systems, Houghton Mifflin, 1978.

11. Smith, J. D., Vibration Measurement and Analysis, Butterworths, 1989.

12. Pavelko V. Some problems of dynamics of mechanical systems at the nonclassic internal and external constraints// The article in this issue of RTU Proceedings.- 2006, pp.105-112. 